\title{
un estudio comparado sobre la petroquímica básica en méxico y argentina entre 1950 y $1990^{\star}$
}

\author{
a comparative study on basic petrochemicals \\ in mexico and argentina between 1950 and 1990
}

\author{
Juan Odisio $\star \star$ \\ Facultad de Ciencias Económicas, Universidad de Buenos Aires, Buenos Aires, Argentina
}

\section{RESUMEN}

Este artículo realiza un estudio comparativo acerca de la evolución de la producción petroquímica en México y Argentina en las décadas de consolidación, auge y abandono de la industrialización sustitutiva de importaciones. Esta industria resultó un eslabón fundamental de dicho modelo económico. En primer lugar, y para comprender mejor el contexto en que dicha industria se desarrolló, se estudia el surgimiento y desenvolvimiento de la petroquímica tanto a nivel mundial como en el ámbito latinoamericano. Luego, se recopilan y analizan numerosas variables sobre la evolución sectorial en los dos países objeto de este texto; especial atención se prestan a las referidas a las dimensiones productiva, de mercado y de la balanza externa, como de los seis bienes petroquímicos considerados "básicos", insumos fundamentales para el resto de la industria.Así, se logran identificar tanto las aristas comunes en la estrategia económica de ambos países, como

\begin{abstract}
This article makes a comparative study on the evolution of the petrochemical production in Mexico and Argentina in the decades of consolidation, boom, and abandonment of the substitutive industrialization of imports. This industry was a fundamental link of this economic model. In the first place, and to better understand the context in which the industry was developed, we study the emergence and development of the petrochemical industry both in the world and in Latin America. Then, numerous variables are collected and analyzed on the sectoral evolution in the two countries to which this text refers; special attention is given to those related to the productive, market and external balance dimensions, as of the six petrochemicals goods considered "basic", fundamental inputs for the rest of the industry. Thus, we can identify both the common features in the economic strategy of both countries as well as those character-
\end{abstract}

* Este trabajo es parte de los resultados obtenidos en una estancia de investigación realizada en la División de Estudios de Posgrado de la Facultad de Economía de la Universidad Nacional de México (UNAM), gracias a su Programa de Becas Posdoctorales.

Fecha de recepción: 28 de febrero de 2018; fecha de aceptación: 24 de febrero de 2019.

$\star \star$ Investigador Asistente del CONICET/IIEP-Baires. Profesor adjunto en la Facultad de Ciencias Económicas de la Universidad de Buenos Aires, Argentina. Doctor en Ciencias Sociales.E-mail: odisio@gmail.com 
aquellas características que distinguieron el devenir de la petroquímica mexicana respecto de la argentina entre 1950 y 1990.

Palabras-clave: Petroquímica. Regulación estatal. Industrialización sustitutiva de importaciones. Desarrollo económico. Empresas públicas. istics that distinguished the evolution of the Mexican petrochemicals from the Argentine between 1950 and 1990 .

Keywords: Petrochemicals. State regulation. Import substitution industrialization. Economic development. Public enterprises.

\section{Introducción: características de la industria petroquímica}

La producción petroquímica fue heredera directa de dos tipos de tecnologías diferentes: la petrolera, interesada en obtener elevados volúmenes de producción y la química orgánica, que buscaba sintetizar moléculas específicas con alto grado de pureza (Chapman, 1991). Desde sus comienzos en la década de 1920, las características técnicas del sector indicaban que los costos laborales eran reducidos en relación al de los insumos, que el proceso productivo se hallaba sujeto a fuertes economías de escala y que la expansión se producía a saltos ${ }^{1}$. Desde el punto de vista de las inversiones funcionaban fuertes barreras al ingreso, por las características de la petroquímica como una industria capital intensiva con rendimientos crecientes a escala, de procesos continuos y automatizados en plantas que debían operar por sobre el $70 \%$ de su capacidad para ser rentables y con una marcada tendencia a la integración vertical en el desarrollo de procesos y productos ${ }^{2}$. La industria presentaba múltiples incentivos para avanzar en una alta integración técnica (Fan, 2000, p. 7). En sentido vertical: economías de escala y aglomeración, presencia de co-productos en los procesos más usuales, importantes costos y riesgos

1 "El costo de este tipo de plantas petroquímicas no deriva únicamente del tamaño de éstas, sino de los materiales de construcción necesarios para evitar la corrosión prematura y reducir el riesgo de accidentes y de la automatización computarizada de los sistemas de control necesarios para la obtención de la calidad requerida" (Rodríguez Magallanes, 2008, p. 25).

2 Los resultados en la petroquímica eran muy sensibles al uso de la capacidad instalada, por lo que su competitividad dependía de mantener un elevado crecimiento de la producción. La operación al 75\% de capacidad incrementaba los costos en más del $10 \%$ en relación con un uso completo de las instalaciones, mientras que afrontar una escala operativa del $50 \%$ implicaba costos unitarios hasta un tercio más elevados (Auty, 1984, p. 330). 
de transporte en productos e insumos, altos costos de transacción, posibilidad de fijar precios de transferencia y aparición de distorsiones en los mercados "aguas arriba". En sentido horizontal se daba especialmente por economías de escala en las actividades de investigación y desarrollo, comercialización, administración y financiamiento del sector (Chudnovsky; López, 1997, p. 14). Los conocimientos tecnológicos involucrados en la industria se desenvolvieron en tres sentidos: tecnología necesaria para evaluar, diseñar y construir un complejo petroquímico; tecnología para la operación del proyecto y la comercialización de su producción; tecnología necesaria para evitar la obsolescencia del proyecto debido al progreso técnico (Khanna, 1984, p. 1.321).

La producción de los insumos petroquímicos era más sensible a las economías de escala que los bienes finales, lo que explicaba que aquellos países con mercados menos desarrollados iniciaran la producción por el final de la cadena, hasta que eventualmente el propio crecimiento justificara la instalación de plantas "aguas arriba", de materias primas petroquímicas (Stobaugh, 1971a, p. 47). Más en general, después de la Segunda Guerra Mundial se multiplicaron los gigantescos complejos integrados; la competencia desatada en el mercado llevó que las mayores firmas petroleras del mundo se interesaran en el negocio, dominado hasta finales de la década de 1950 por las grandes empresas químicas. Ello condujo en definitiva a una marcada tendencia hacia la integración vertical del sector:

En la década de 1960, cuando las plantas se estaban volviendo más grandes e intensivas en capital y nuevas tecnologías proveían enormes ganancias de productividad, las firmas petroleras se vieron a sí mismas teniendo una ventaja sobre las firmas químicas más viejas con plantas más pequeñas y obsoletas. Las compañías petroleras además enfrentaron pocas dificultades en encontrar acceso a la tecnología petroquímica. Apareció una serie de nuevas plantas controladas por trasnacionales petroleras. (Khanna, 1984, p. 1.323)

La integración demandaba grandes inversiones, pero el avance en la cadena petroquímica implicaba obtener bienes de creciente valorización en cada eslabón productivo (Ilustración 1) 3 $^{3}$.Así, las refinerías emplazaron

3 Por ejemplo, hacia 1967 el benceno estadounidense tenía un valor medio de 5,7 
plantas petroquímicas comunicadas con sus instalaciones, ampliando su campo de operaciones en un mercado en gran expansión y que permitía una mayor rentabilidad para sus mercancías; mientras que la industria química, frente a la creciente demanda de subproductos hidrocarburíferos, avanzó progresivamente también en la obtención de sus propios insumos por medio del craqueo (Berti, 1963, p. 7-8). Asimismo, fue práctica usual la firma de convenios entre varias firmas petroquímicas, por el que mediando la instalación de una refinería conjunta, se aseguraban la provisión de materias primas y conformaron grandes complejos petroquímicos (Guglielmo, 1960, p. 38).A grandes rasgos, las empresas petroleras pasaron a dominar la oferta de petroquímicos básicos e intermedios, mientras las químicas se especializaron en los bienes finales.

Ilustración 1 -Valor (teórico) agregado por la petroquímica (mediados década de 1960)^

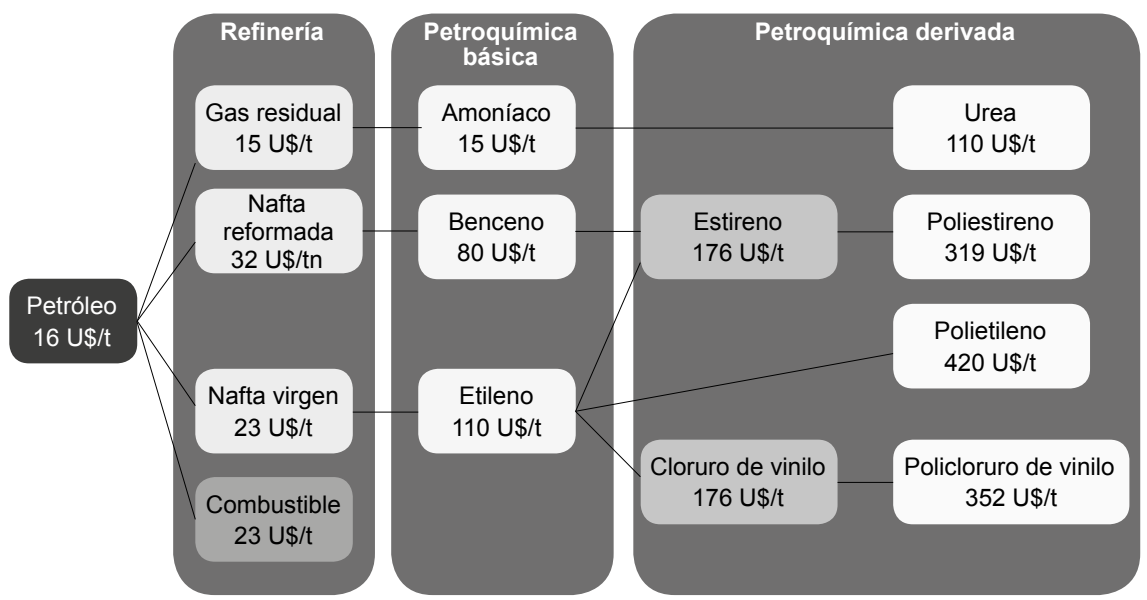

Fuente: elaboración propia en base a CEPAL (1966, p. 17).

* Los valores correspondientes a cada producto corresponden a un esquema "teórico" elaborado por la CEPAL en 1966 considerando una "situación hipotética de una refinería de petróleo moderna, de capacidad razonable y dotada de un conjunto de unidades adecuadas para el mercado tipo de un país latinoamericano".

Un elemento adicional tendiente a la integración era que como los insumos petroleros son compuestos de hidrocarburos, y como los pro-

veces su materia prima, mientras que el precio del fenol o el dodecilbenceno (dos de sus derivados), representaban algo más de 17 veces el costo de su insumo (Grioni, 1968, p. 5-9). 
cesos de separación resultaban escasamente selectivos, además del producto petroquímico principal se podían obtener numerosos sub-productos de la misma corriente de refinación, que debían ser aprovechados para maximizar la rentabilidad de las instalaciones (Khanna, 1984, p. 1.320). Por tales motivos, tanto técnica y económicamente, lo más conveniente era ubicar las instalaciones petroquímicas en la cercanía de las instalaciones que proveían las materias primas. En caso contrario, la construcción de los ductos de transporte necesarios implicaba un incremento sustancial sobre los costos de inversión a amortizar ${ }^{4}$.

En los países subdesarrollados - como en Latinoamérica - los impactos que la petroquímica tenía sobre la economía local se venían amplificados, y de allí el interés que los respectivos gobiernos colocaron en su fomento en los años de la industrialización sustitutiva, como veremos más abajo. De una parte, mediante la obtención de fertilizantes, plaguicidas y otros agroquímicos, permitía incrementar los rendimientos agrícolas.Además, aportaba un elevado valor agregado sobre sus materias primas, impulsando al proceso de industrialización. Proporcionaba también - en línea con los objetivos de la política económica de la posguerra - una manera eficiente de ahorrar divisas, tanto por mayor sustitución de bienes importados como por la posibilidad de generar nuevas ventas externas provenientes del sector industrial. En suma, la importancia que encontraba en la industria petroquímica se puede resumir en cinco funciones principales: aporte a la tecnificación de la producción agrícola; incremento de la productividad en el sector industrial; profundización de la sustitución de importaciones; diversificación de la canasta exportadora y; aumento de los niveles de empleo manufacturero y del ingreso nacional (Chávez Aguilar, 1974, p. 25-26).

\section{La industria petroquímica en el mundo}

La petroquímica moderna nació a principios de la década de 1920 , asociando los avances de la industria química con los de la petrolera, al

4 Si bien para algunos productos - especialmente aquellos de los eslabones finales de la cadena - se podían instalar cerca de los centros consumidores, la lógica que primaba era la de localizar las plantas cerca de las refinerías (Chávez Aguilar, 1974, p. 33-34). 
demandar derivados del petróleo y el gas natural para obtener nuevos subproductos diferentes de los combustibles, lubricantes, ceras u otras manufacturas petroleras. Las mejoras introducidas en el proceso de refinación proporcionaron las materias primas necesarias para su despegue, en abundancia y a bajo costo (Speight, 2005). El gas natural y el petróleo crudo fueron las fuentes de hidrocarburos intermedios que la industria petroquímica utilizaba como materias primas básicas para sus tres cadenas productivas principales: olefinas, aromáticos y metanol ${ }^{5}$.

La supremacía inicial de Estados Unidos frente a Europa y Japón en el desarrollo de métodos de destilación probablemente se puede explicarse con referencia a sus mayores requerimientos de naftas por unidad de crudo (Grioni, 1968). Desde 1920, cuando la Standard Oil instaló la primera planta petroquímica con capacidad comercial en South Charleston (Virginia Occidental) hasta los años de la Segunda Guerra Mundial, los esfuerzos se ligaron sólidamente al despliegue de la industria automotriz. En los años de entreguerras otras grandes empresas realizaron adelantos productivos con los petroquímicos fundamentales: Union Carbide y Dow Chemical desarrollaron derivados del etileno para la industria plástica y textil mientras Shell comenzó a elaborar amoníaco, base de fertilizantes y explosivos (Garritz Cruz, 1996, p. 35). En el continente europeo, la ausencia de reservas importantes hidrocarburíferas retardaron a la industria. La posibilidad de contar con carbón, cuya química estaba en pleno auge, permitía obtener prácticamente todos los productos que producía la petroquímica estadounidense pero por vía carboquímica, técnica particularmente adelantada en Alemania y la Unión Soviética (Guglielmo, 1960, p. 9) $)^{6}$.

5 "Del gas natural se recuperan el etano y los licuables del gas para la producción de olefinas y diolefinas; además algunos químicos importantes como el metanol y el amoníaco se producen a partir del metano obtenido del gas natural. Por otra parte, del petróleo se utilizan algunos gases de refinería provenientes de diferentes procesos de la refinación para la producción de olefinas y gases licuables; utilizando procesos de cracking y reformado, algunos destilados y residuos del crudo son precursores de olefinas y aromáticos.” (Rodríguez Magallanes, 2008, p. 24)

6 Inicialmente, además la carboquímica tenía como ventaja que la mezcla de aromáticos que arrojaba tenía una gran proporción de benceno (mucho más que los métodos de reformación del petróleo), que era por entonces el insumo aromático más demandado. Otras técnicas también permitían obtener subproductos de la petroquímica utilizando otras materias, como el azúcar (sucroquímica), la carbonización de la madera o la fermentación alcohólica. 
La conflagración mundial implicó un fuerte impulso a la elaboración y obtención de nuevos derivados hidrocarburíferos reclamados por el esfuerzo bélico, tales como naftas especiales, explosivos (tolueno), caucho sintético, nilón, polietileno y solventes. Si bien en esta época la fabricación petroquímica se inició en algunos otros países - concretamente: Canadá, Inglaterra y Rusia -, Estados Unidos alcanzó un nivel de desarrollo muy superior. Sólo entre 1942 y 1945 la producción petroquímica norteamericana total pasó de 230 mil toneladas a 1,4 millones. El énfasis primordial se ubicó en la fabricación de caucho sintético. Como parte central de las necesidades de la guerra, el Estado impulsó fuertemente o se hizo cargo de manera directa, de la promoción, construcción y explotación de nuevas plantas, además de asegurar una elevada demanda a las previamente existentes (Instituto Venezolano de Petroquímica, 1964, p. 3).

Por otra parte, dicho arresto, con sus requerimientos para trasladar rápidamente suministros a regiones alejadas de los centros de producción, sentó las bases para el desarrollo de novedosos métodos de transporte, almacenamiento y operación de materiales petroquímicos. Los mismos permitieron alcanzar grandes economías de costos con los movimientos en masa o a granel de estos bienes y proporcionó la infraestructura y los medios elementales para establecer, más tarde, un activo comercio internacional (Berti, 1963, p. 67).

Si bien hasta entrada la década de 1950 el ritmo de expansión de la producción petroquímica se desaceleró en los Estados Unidos - en relación con el vertiginoso incremento del lapso bélico -, siguió siendo muy importante. La mayor expansión de la demanda por combustibles frente a la del acero, motivó que los adelantos técnicos en la refinación - y por ende, de la petroquímica - superaran ampliamente a los de la química de la carbonización, explicando en parte la supremacía norteamericana y el rezago europeo. Por su parte, en el Viejo Continente la petroquímica no reaccionó de inmediato tras la guerra: a la necesidad de reparación de las refinerías, fuertemente castigadas en la contienda, se le sumaba el hecho de que los métodos carboquímicos seguían siendo preferidos.A diferencia de lo que sucedía en Estados Unidos, la siderurgia se encontraba (relativamente) más desarrollada que la industria petrolera.

Finalmente, la estabilización y reconstrucción económica y el rearme por la guerra fría favorecieron un despliegue más vigoroso de la industria, 
surgiendo en Europa el objetivo de equiparar su desarrollo al alcanzado en los Estados Unidos. También jugó un papel importante la mayor disponibilidad de insumos petrolíferos a escala mundial, con la puesta en producción de los campos petroleros de Oriente Medio (Doan, 1967, p. 35). Por ejemplo, en Inglaterra y Alemania a finales de la década de 1940 los químicos orgánicos derivados de materias primas petroquímicas representaban sólo el 10\%-15\% del total del sector, mientras que para los primeros años de la década de 1960 superaban el 60\% (CEPAL, 1966, p. 13).

Con relación a las materias primas, en Estados Unidos y Canadá la mayor demanda de cortes de naftas y la amplia disponibilidad de gas (tanto natural como derivado de la refinación del crudo, o sea, residual y LPG) llevaron a que, en términos generales, su desarrollo petroquímico se orientara hacia el aprovechamiento de dicho insumo. Mientras tanto, en Europa la refinación de crudos livianos procedentes de África y el Oriente Medio con alto contenido de naftas y una demanda de combustibles menor a la estadounidense, permitieron la obtención de excedentes de naftas, por lo que en la petroquímica europea - como asimismo la japonesa - tendió a orientarse hacia la producción de aromáticos (Grioni, 1968, p. 3).

Con todo, hacia 1959 Estados Unidos aun poseía el 90\% de la capacidad instalada petroquímica a nivel global, junto con "las materias primas de menor costo, la energía más barata y la tecnología líder en el mundo" (Doan, 1967, p. 35). Sin embargo, en un lustro todo eso cambiaría con el crecimiento de la competencia tecnológica y productiva, sobre todo - pero no exclusivamente - de Europa y Japón. A mediados de la década de 1960, la capacidad productiva de la petroquímica estadounidense había caído a menos de la mitad del total mundial. Fuera del mundo desarrollado el avance de la petroquímica solía circunscribirse, con algunas tímidas excepciones, a la obtención de abonos nitrogenados mediante la separación del hidrógeno contenido en el gas natural (Guglielmo, 1960, p. 5).

El sector era de los de más rápido crecimiento: su evolución se encontraba muy asociada al crecimiento de la población y los ingresos. Al crecer el producto total de un país, la evolución tanto de la producción como de las importaciones petroquímicas superaban a los promedios verificados en las demás ramas manufactureras (Cavanna, 1968, p. 4-5). 
La petroquímica se convirtió así en la principal rama de la industria química, siendo uno de los puntales del crecimiento económico durante los lustros de posguerra (Berti, 1963, p. 10) ${ }^{7}$. Como se advierte en la Tabla 1, la industria creció a nivel mundial en promedio un $15 \%$ acumulativo al año entre 1950 y $1970^{8}$.

Tabla 1 - Producción petroquímica (millones de toneladas) por regiones

\begin{tabular}{l|r|r|c}
\multicolumn{1}{c|}{ REGIÓN } & 1950 & 1970 & CREC. ANUAL PROMEDIO \\
\hline Estados Unidos y Canadá & 3 & 23,5 & $11 \%$ \\
\hline Europa Occidental & 0,5 & 19,5 & $20 \%$ \\
\hline Japón & - & 9 & - \\
\hline URSS y Europa Oriental & - & 5,5 & $14 \%$ \\
\hline Resto del mundo & 0,2 & 3 & $15 \%$ \\
\hline Total & 3,7 & 60,5 & \\
\hline
\end{tabular}

Fuente: elaboración propia en base a datos de Garritz Cruz (1996, p. 37).

En términos generales, la mayor competencia y el rápido crecimiento del mercado en esos años desató una "carrera inversa" entre niveles de producción y evolución de los precios petroquímicos, que además de motorizar un fuerte proceso de expansión productiva gracias a la creciente demanda, impuso un importante acicate para el avance tecnológico en el sector. Éste estuvo liderado por los principales países productores: un análisis de 53 productos petroquímicos (plásticos, cauchos y fibras) a principios de la década de 1970, indicaba que 46 habían sido desarrollados por empresas de los Estados Unidos o Alemania, siendo conseguidos los siete restantes en Inglaterra e Italia (Stobaugh, 1971a, p. 44).

A favor de esa rápida evolución, debe remarcarse el impulso evidente que provino desde la demanda por el aumento en el consumo de nuevos

7 Desde principios de la década de 1950 la petroquímica en la OCDE se expandió a una tasa que duplicaba el crecimiento del producto bruto en cada país y en la década de 1960 atrajo, en ese grupo de naciones, un quinto del total de la inversión industrial (Auty, 1984, p. 328).

8 Como explicaba una tesis de finales de la década de 1960: "Los productos químicos orgánicos representan casi el $70 \%$ del tonelaje total de la producción mundial en la industria química. El 85\% de los productos químicos orgánicos se producen a partir del petróleo y del gas natural", lo que implicaba que el 60\% del total de la producción de la rama química correspondía a la petroquímica, siendo incluso que dicho porcentaje tendía a aumentar (García Lizama, 1968, p. 20). 
productos sintéticos - cauchos, jabones, colorantes, plásticos, fibras -, al avanzar la sustitución de anteriores productos naturales o de derivados orgánicos o la directa aparición de nuevos materiales artificiales, más eficientes y económicos. De tal modo, la amplia diversificación de la demanda permitió por ese entonces que la petroquímica perdiera su estrecha ligazón que la unía a la industria automotriz desde sus orígenes para pasar a ser utilizada por un vasto espectro de sectores intermedios y de consumo final: la agricultura (fertilizantes y plaguicidas), plásticos y resinas, fibras y cauchos sintéticos, detergentes, solventes, explosivos y productos farmacéuticos se ubicaron entre los usos principales. El tamaño del mercado era, en definitiva, la cuestión determinante para la expansión petroquímica?

Hacia mediados de la década de 1960 Estados Unidos mantenía su predominio, especialmente visible con relación a los niveles de producción, aunque - como señalamos - iba en declive ${ }^{10}$. El más tardío desarrollo de la petroquímica europea permitió que se comenzara la producción contando con los últimos adelantos tecnológicos, muy diversificada y ubicándose rápidamente en ramas que, en más de un caso, incluso aventajaban a sus pares norteamericanas (Instituto Venezolano de Petroquímica, 1964, p. 5).Además, como siguieron apareciendo nuevos productos y nuevas aplicaciones en distintas ramas industriales, la petroquímica europea entró en directa competencia por el desarrollo de mercados y tonelaje de producción con la industria de los Estados Unidos. Por otra parte, este país mantuvo una ventaja importante por

9 Los motivos por los cuales las grandes compañías optaban por introducir nuevos productos petroquímicos en los mayores mercados fueron resumidos del siguiente modo: "Una comunicación cercana entre la planta y los clientes es necesaria. Además, el productor desea no depender del mercado de exportación para una porción demasiado grande de su capacidad instalada. En adición, dada una relativamente baja elasticidad precio de la demanda del producto, el productor tiene pocos incentivos a buscar una locación con costos menores, en especial porque probablemente espera que no sean radicalmente diferente de los vigentes en su país de origen" (Stobaugh, 1971a, p. 46).

10 Señalaba el por entonces presidente de Dow Chemical que, en tanto los productos químicos constituían el mayor contribuyente al balance comercial de los Estados Unidos, era necesario emprender un nuevo esfuerzo para mejorar la competitividad de los productos petroquímicos, revelando indirectamente con ello la pérdida de posiciones relativas de la industria estadounidense en los mercados internacionales (Doan, 1967, p. 36). 
contar con un mercado más homogéneo y de tamaño continental a disposición, como por utilizar el gas natural disponible en su territorio como principal insumo petroquímico. Es decir que la industria estadounidense se desarrolló más orientada hacia adentro, en lo tocante a sus materias primas como al mercado principal de sus productos (Auty, 1984, p. 329). En Europa la mayor dependencia del comercio internacional se mostraría luego como una debilidad para las firmas petroquímicas.

Por otra parte, el fortalecimiento de la industria en la Unión Soviética y el acelerado impulso que tomó en Japón en la misma época conformaron los casos de crecimiento más notable ${ }^{11}$. Pero también en la década de 1960 - y de manera muy perceptible, en la década siguiente - se amplió el peso de la petroquímica en los países periféricos de desarrollo industrial intermedio, tales como Egipto o las naciones latinoamericanas que poseían los mayores mercados domésticos para productos petroquímicos: Argentina, Brasil o México, como veremos en el siguiente apartado.

Con todo, a mediados de la década de 1970 comenzó a cambiar el funcionamiento de la industria a nivel global. Si bien la petroquímica se vería severamente afectada por los sucesivos shocks petroleros, en realidad su crecimiento se había comenzado a debilitar desde antes (Auty, 1984, p. 329). El alza y, especialmente, la volatilidad en el precio de las materias primas implicó presiones importantes sobre la evolución de la industria ${ }^{12}$. La rentabilidad se vio disminuida y afectó marcadamente a los países europeos y a Japón que no contaban con yacimientos de importancia, mientras que los estadounidenses, que se habían enfocado en el aprovechamiento del gas disponible en el país, sufrieron menos la crisis en un primer momento.

Tras un alza inicial luego del primer shock petrolero, y más allá de las presiones que debían afrontar por los costos en crecimiento, durante la década de 1980 se produjo un derrumbe de los precios petroquímicos

11 El caso japonés (y en menor medida, también el brasileño) es paradigmático de la capacidad de agregación de valor de la petroquímica (que describimos más arriba); la industria se desarrolló ampliamente en ese país incluso careciendo casi por completo de materias primas básicas.

12 Por ejemplo, entre 1972 y 1981 la incidencia de los insumos pasó de representar el $50 \%$ al $80 \%$ sobre el costo total de aquellas plantas que utilizaban nafta. En paralelo, los costos relativos al capital descendieron del 36\% al 16\% (Khanna, 1984, p. 1.320). 
en los mercados internacionales ${ }^{13}$. Dicha retracción obedeció a fuerzas actuantes tanto del lado de la oferta como de la demanda: la sobreoferta de productos petroquímicos en los mercados mundiales fue resultado del estancamiento económico, no previsto en el mundo desarrollado, donde las grandes empresas habían realizado enormes inversiones a principios de la década de 1970 que no encontraron demanda solvente en los años siguientes. Pero asimismo fue ocasionado por la aparición de enormes establecimientos productivos en Medio Oriente ${ }^{14}$.

Frente a ello, la estrategia adoptada por los mayores jugadores a nivel mundial implicó una acción en varios frentes: por un lado, se produjo una mayor concentración empresaria, buscando reducir la incertidumbre y ganar control sobre los precios de los productos petroquímicos ${ }^{15}$. Este proceso implicó la compra, fusión y desaparición de numerosas plantas y firmas en el sector. Por otra parte, estos productores más avanzados buscaron especializarse en los bienes finales de la cadena, de menor escala de producción y alto valor unitario, a fin de protegerse de la caída que sufrían los precios de los plásticos, fibras y elástomeros de procesos estandarizados y gran volumen (Khanna, 1984, p. 1.323). En adición a la concentración y la diversificación, las grandes empresas emprendieron una agresiva estrategia de penetración de los mercados internacionales, empujando los precios cada vez más hacia la baja ${ }^{16}$.

A nivel global, el mercado petroquímico se sumió en una importante depresión en la que los principales perjudicados resultaron ser los pro-

13 El crecimiento en los precios internacionales del etileno, el petroquímico básico más difundido, de la mitad del mostrado por el petróleo crudo; mientras que para los bienes finales fue, como mucho, de un cuarto (Auty, 1984, p. 329).

La estrategia permitió a estos nuevos productores desplazar en parte importante a los grandes jugadores que, hasta entonces, habían dominado el mercado mundial: los "ingresantes de las economías ricas en minerales son capaces de modificar los precios para capturar suficientes ventas para garantizar la operación a máxima capacidad. Esto puede ser hecho descontando los precios de los insumos y, si es necesario, subsidiando los insumos de capital. Ambas medidas son facilitadas donde, como en muchos países en desarrollo ricos en petróleo, el Estado es el oferente tanto de los insumos y del capital con reducidos costos de oportunidad" (Auty, 1984, p. 331).

15 Un interesante análisis del proceso de concentración de las petroquímicas estadounidenses tras el incremento de precios petroleros de la década de 1970 partiendo desde la teoría de los costos de transacción puede verse en Fan (2000).

16 Historia de una empresa nacional. Petroquímica General Mosconi, Todo es Historia, p. 11, mayo 1981, Suplemento Especial. 
ductores japoneses y europeos ${ }^{17}$. La sensible elevación en los costos, al no poder mantenerse los niveles de producción, y la caída en la demanda, dieron origen a una profunda crisis que duró hasta $1987^{18}$. A partir de allí comenzaron a verse algunos atisbos de recuperación, aunque no se lograron reproducir las elevadas y generalizadas tasas de crecimiento de las décadas anteriores.

\section{La petroquímica latinoamericana}

En Argentina y México se dieron los primeros avances de la industria a nivel latinoamericano durante la década de 1940, en el contexto de la Segunda Guerra Mundial. Poco después, en la década siguiente, en el continente la petroquímica brasileña había tomado el primer lugar, y resultaba "la más avanzada y diversificada en el grupo de los países subdesarrollados" (Guglielmo, 1960, p. 68). Pero a principios de la década de 1960, México tomó indiscutidamente el primer puesto, a la vez que apareció una producción de relativa relevancia en Colombia, Perú y Venezuela (Instituto Venezolano de Petroquímica, 1964).

Además de ser los países económicamente más avanzados del subcontinente, en Argentina, Brasil y México era donde las petroleras locales (públicas y privadas) tenían el papel preponderante en la actividad de refinación, mientras que en Perú,Venezuela y Colombia las empresas extranjeras tenían preeminencia. Resulta asimismo sugestivo indicar que, de esos tres países, en Colombia -donde las compañías nacionales eran relativamente más importantes - fue donde más avanzó la petroquímica durante la década de 1960 (Firpo, 1968). Particularmente, se notaba un mayor énfasis en el avance de la producción petroquímica cuando

17 Como resultado de la última década Europa y Japón se achican, Temas, año 11, n. 35, 1986.

18 La industria petroquímica tenía ciclos de auge-recesión bastante marcados de ocho años, relacionados al modo en que se incrementaba la capacidad productiva: "Para ser competitivo a nivel mundial, es necesario construir plantas que representan entre el 1\% y el $3 \%$ de la demanda mundial total; por lo tanto, si en un corto período de tiempo se construyen dos o tres plantas de estas características, el equilibrio oferta/ demanda del mercado se verá afectado, obligando a los productores a disminuir los precios de venta y aquellos productores menos eficientes tenderán a desaparecer" (Rodríguez Magallanes, 2008, p. 35). 
empresas estatales estaban al frente de la actividad petrolera en cada nación (CEPAL, 1966, p. 24-26). En cada caso, el impulso debía asociarse a un amplio esfuerzo coordinado desde el Estado:

[La] localización geográfica de grandes complejos industriales, requiere la materialización de planes de infraestructuras (carreteras, ferrocarriles, puertos, energía eléctrica, etc.) y urbanización, sin los cuales se afectaría fuertemente la evolución de dichos complejos. De aquí que en América Latina la promoción del desarrollo de la petroquímica sólo puede ser encarada por los gobiernos en el contexto de una planificación global de las economías nacionales o regionales. (CEPAL, 1966, p. 14)

Como prueba del avance latinoamericano en el sector, se puede mencionar que en un seminario sobre la industria química organizado por CEPAL en Caracas durante 1963, al tratarse la petroquímica, se puso de manifiesto el interés de industriales y organismos de planificación sobre las posibilidades de su desarrollo, como asimismo la inopia de informes y la escasa comparabilidad de la información presentada por cada país. Para 1965, había iniciado actividades petroquímicas también Chile. Ese año, con casi 220 millones de dólares, la producción conjunta de los países latinoamericanos alcanzó a decuplicar el valor obtenido en 1959 y se esperaba que para 1970 lo superara en más de 15 veces $^{19}$. Otro informe de la CEPAL indicaba que si bien el sector se encontraba rezagado en Latinoamérica, tenía un amplio espectro de oportunidades para avanzar rápidamente, siguiendo la misma evolución que antes se había visto en Europa; esto es, pasando de métodos carboquímicos tradicionales a adoptar la técnica de la moderna petroquímica, y desde los bienes finales hacia las materias primas:

En América Latina, todavía es incipiente el adelanto de las industrias químicas derivadas del petróleo y del gas natural. Al contrario de lo que ocurre en la mayoría de los países más industrializados, se ha comprobado que en América Latina, por ahora casi las dos terceras partes de la producción química latinoamericana corresponden a la industria química ligera, producción de bienes de consumo y formulaciones o mezclas, como pin-

19 Sobre el valor conjunto de 1965, Argentina aportaba el 14\% del total, Brasil el 28\% y un 37\% México (Firpo, 1968). 
turas, productos de limpieza y tocador, detergentes formulados, etc. (CEPAL, 1965, p. 2)

Por su parte, los "sectores dirigentes" comenzaban a plantear la posibilidad de avanzar con la integración industrial y la complementación económica a nivel regional. Por otra parte, también se evidenció la confusión existente en cada país en torno a la delimitación de las actividades petroquímicas específicamente (respecto de la industria química o la petrolera), lo que dificultaba tanto el establecimiento de una correcta legislación de promoción, como la determinación de la participación que le correspondía, en cada caso, al sector privado o público (CEPAL, 1966, p. v-vi).

Como también sucedía en otros renglones industriales, la Asociación Latinoamericana de Libre Comercio (ALALC, creada en 1960) había permitido un efimero auge del comercio regional. A pesar de la posibilidad potencial de alcanzar mejoras de escala, la lógica de promoción en cada país había actuado en contrario. Como marcaba un observador argentino con participación en la industria, hacia finales de la década de 1960 ya no se guardaba demasiada esperanza en torno a la posibilidad de un fuerte avance de la integración productiva entre los miembros de la Asociación:

Los sucesos internacionales han endurecido las posiciones de cada uno de los países y es evidente que ninguno desea renunciar a sus producciones básicas. El problema que esto plantea a los países menores es insoluble por el momento, a pesar de las declaraciones diplomáticas.

Las legislaciones van otorgando más y más promoción a las inversiones básicas en todos los países de la ALALC y ello hace que vayan encontrando posibilidades, producciones más y más marginales. En los grandes parece invertirse la tendencia, y habiéndose definido que no hay que acelerar artificialmente la radicación de las industrias, los Gobiernos van ahora hacia la mayor eficiencia.

$[\ldots]$

Es previsible esperar que la influencia estatal en la legislación sobre el sector petroquímico, especialmente en lo que a promoción se refiere, se vaya acentuando.

Lo que no parece factible es que en sus legislaciones los Gobiernos contemplen las recomendaciones emanadas por la ALALC. 
Los proyectos de integración que formule la CADI [Comisión Asesora de Desarrollo Industrial, creada en el seno de la ALALC en 1963], y que se encuentran muy avanzados en su formulación teórica, carecen por ahora de aplicabilidad. (Firpo, 1968, p. 13-17)

Como dijimos, en todos los países del mundo el Estado jugó un papel de primer orden en el despliegue de la industria petroquímica en las décadas que siguieron a la Segunda Guerra Mundial. Su injerencia en el desarrollo de nuevas plantas y la planificación del sector se hallaba asociado - en gran parte - al papel que cumplía en la provisión del petróleo (Berti, 1963, p. 8). En los países más atrasados también resultó central el papel de la planificación, por la necesidad de materializar una importante infraestructura que permitiera el despliegue tanto de la rama específica como del resto del sector industrial, como asimismo por la exigencia - ya señalada - de inmovilizar grandes masas de capital, lo que dejaba a las petroleras del sector público como uno de los pocos, si no los únicos, posibles inversores locales ${ }^{20}$. Asociado a ello, también se verificó un cambio en la estrategia de las grandes empresas trasnacionales de Estados Unidos y Europa en la década de 1960; tras una primera expansión de la inversión directa hacia países menos desarrollados se fueron mostrando más inclinadas a fortalecerse en sus mercados domésticos y exportar al resto del mundo las patentes y la tecnología (Stobaugh, 1971a, 1971b).

Por otra parte, atentaba contra el rápido desarrollo de la petroquímica en Latinoamérica el que, todavía en la década de 1960, la estructura de la industria petrolera no permitía obtener grandes cantidades de materias primas petroquímicas (Tabla 2), vinculadas a los métodos catalíticos que eran los que permitían obtener mayor contenido de petroquímicos básicos. En relación con los principales métodos de refinación, la caída en la demanda de cortes de combustibles pesados (como el fuel oil) y el incremento en el consumo de derivados petroquímicos impondrían un proceso de ampliación y reconversión de las unidades de reformación desde la destilación atmosférica hacia los procesos catalíticos ${ }^{21}$.

\footnotetext{
20 Aunque en desventaja frente a las grandes firmas multinacionales (CEPAL, 1966, p. 14-25).

21 Un completo repaso técnico acerca de los sucesivos procesos de destilación, reformación y craqueo puede consultarse en (Rodríguez Magallanes, 2008, cap. 2) o resumidos en (Chow Pangtay, 2002, Cuadro 3).
} 
Tabla 2 - Capacidad de refinación, países seleccionados, 1964

\begin{tabular}{|c|c|c|c|c|c|c|}
\hline \multirow[t]{2}{*}{ PAís } & \multirow[t]{2}{*}{ REFINERÍAS } & \multirow{2}{*}{$\begin{array}{l}\text { CAPACIDAD } \\
\text { TOTAL }\end{array}$} & \multicolumn{2}{|c|}{ Craqueo catalítico } & \multicolumn{2}{|c|}{ REFORMACIÓN CATALÍTICA } \\
\hline & & & $\begin{array}{c}\text { BARRILES } \\
\text { DIARIOS }\end{array}$ & $\begin{array}{c}\text { PORCENTAJE } \\
\text { S/TOTAL }\end{array}$ & $\begin{array}{c}\text { BARRILES } \\
\text { DIARIOS }\end{array}$ & $\begin{array}{c}\text { Porcentaje } \\
\text { S/TOTAL }\end{array}$ \\
\hline Argentina & 13 & 372.700 & 58.500 & $15,7 \%$ & - & - \\
\hline Brasil & 9 & 298.900 & 47.500 & $15,9 \%$ & 21.500 & $7,2 \%$ \\
\hline México & 6 & 419.000 & 64.000 & $15,3 \%$ & 32.000 & $7,6 \%$ \\
\hline Venezuela & 15 & 1.070 .500 & 44.200 & $4,1 \%$ & 30.600 & $2,9 \%$ \\
\hline Latinoamérica & 69 & 2.547 .500 & 296.200 & $11,6 \%$ & 107.600 & $4,2 \%$ \\
\hline
\end{tabular}

Fuente: elaboración propia en base a CEPAL (1965, Cuadro 3).

Como señalamos más arriba, por sus características técnicas la petroquímica demandaba un desarrollo extremadamente concentrado. Pero en ausencia de grandes empresas con elevados niveles de escala en el medio local, la producción encontraba dificultades para desenvolverse. Quedaban como actores casi exclusivos las grandes empresas industriales del Estado o bien los capitales extranjeros. Por otra parte, la suerte de la cadena dependía en medida importante de la posibilidad de colocar los subproductos de la cadena, es decir, de la demanda proveniente de los encadenamientos. En ese sentido, en los países latinoamericanos de industrialización más avanzada, los inicios de la petroquímica estuvieron asociados con la promoción de la industrialización "pesada", que pretendía dinamizar el conjunto de sectores productores de bienes de capital e insumos industriales de uso difundido, como la petroquímica.

Ahora bien, la estrechez del mercado local, la inestabilidad política, la poca profundidad de los mercados financieros, la inexperiencia en el manejo de nuevas tecnologías, entre otros límites, indicaban que el impulso para favorecer el surgimiento de estos nuevos sectores, intensivos en capital y tecnología, debía basarse primordialmente en el apoyo del Estado. Al desconocimiento técnico, se le sumaba la reducida dimensión de las primeras plantas que implicaba producir a costos superiores a los vigentes en los mercados internacionales. Por eso durante los "años dorados" del capitalismo de posguerra, los gobiernos en distintos países del continente buscaron atacar algunos de estos problemas, con diferentes medidas y resultados. Así, aparecería el énfasis en lograr la integración vertical, el desarrollo completo de la cadena productiva y la implantación de grandes empresas de escala mundial.

Como puede verse en la Tabla 3, la capacidad instalada en los tres 
países más industrializados del continente, en muchos casos, no llegaba al mínimo eficiente. Las estimaciones de la CEPAL, tomando como parámetro las condiciones de producción vigentes en el mundo industrializado, permitían dar cuenta de las deseconomías de escala que operaban en la industria latinoamericana. El polietileno era el único artículo petroquímico que encontraba niveles de producción en Argentina, Brasil y México que superaban el mínimo eficiente. De allí, que el organismo asociara el futuro del sector con la necesidad de integración económica:

Las características inherentes a la petroquímica en lo que se refiere al monto de los capitales requeridos, llevan a conceder especial atención al hecho de que sólo pocos países - y para reducido número de productos presentaran a mediano plazo demandas que permitan pensar en proyectos de escala económica. Se deduce de ello la conveniencia de adicionar las demandas de varios países, con miras a satisfacerlas a través de proyectos de niveles considerados rentables y abrir así una vía de realización al proceso de integración latinoamericano. (CEPAL, 1966, p. x)

Tabla 3 - Capacidad instalada total por país y capacidad mínima económica, circa 1965 (en toneladas)

\begin{tabular}{l|c|c|c|c}
\hline \multicolumn{1}{c|}{ Producto } & $\begin{array}{c}\text { EsCALA } \\
\text { EFICIENTE }\end{array}$ & ArgentinA & BrasiL & MÉXICO \\
\hline Etileno & $\mathbf{6 3 . 5 0 0}$ & 23.200 & 33.000 & 72.000 \\
\hline Metanol & $\mathbf{3 3 . 0 0 0}$ & 40.000 & 18.000 & 15.000 \\
\hline Negro de humo & $\mathbf{2 0 . 0 0 0}$ & 25.000 & 33.200 & 15.000 \\
\hline Amoníaco & $\mathbf{6 6 . 0 0 0}$ & - & 99.000 & 140.000 \\
\hline Polietileno & $\mathbf{1 8 . 0 0 0}$ & 20.000 & 28.000 & 18.000 \\
\hline Estireno & $\mathbf{2 0 . 0 0 0}$ & 29.000 & 22.000 & 15.000 \\
\hline Caucho & $\mathbf{4 0 . 0 0 0}$ & 35.000 & 40.000 & 40.000 \\
\hline Dodecilbenceno & $\mathbf{3 8 . 0 0 0}$ & - & - & 38.000 \\
\hline Urea & $\mathbf{6 6 . 0 0 0}$ & - & 75.000 & 103.000 \\
\hline
\end{tabular}

Fuente: elaboración propia en base a CEPAL (1965, Cuadro 10).

Generalizando, la intervención pública sobre la petroquímica se desenvolvió en cinco frentes: la decisión de dirigir "estratégicamente" 
la industria; el otorgamiento de importantes subsidios para fomentar la inversión privada; el establecimiento de elevadas barreras arancelarias y para-arancelarias para proteger al sector local; el mantenimiento de precios deprimidos de las materias primas hidrocarburíferas y; por último, el impulso a la producción mediante la instalación de empresas estatales (Chudnovsky; López; Porta, 1993, p. 29).

De tal suerte, sobrepasando los problemas de los primeros años y con ese fuerte impulso estatal, en la década de 1970 comenzaron a aparecer complejos y plantas petroquímicas en Latinoamérica con niveles de escala y eficiencia cercanos a los vigentes en la frontera tecnológica y productiva internacional (Chudnovsky; López, 1997, p. 18). Pero al mismo tiempo - como ya se señaló - los shocks petroleros cambiaron la lógica de la industria petroquímica, que se vio forzada a buscar nuevas estrategias en todo el mundo. En ese nuevo marco, los últimos esfuerzos del Estado empresario en Latinoamérica chocaron además con el giro de la estrategia económica impuesta después de agosto de 1982. Asediados por la crisis de deuda y el acusado giro global hacia el neoliberalismo, los distintos gobiernos fueron abandonando tanto el concepto que la industrialización compleja era el sendero que permitiría avanzar hacia el desarrollo económico como también las amplias atribuciones que, en ese proceso, se habían adjudicado al Estado y sus empresas.

Para finales de la década los lineamientos económicos habían virado notoriamente en el continente. La apertura económica y la desregulación de los mercados condujeron a un proceso de creciente desindustrialización y privatización de empresas estatales. En ese contexto, los impulsos de los Estados latinoamericanos en pos de una creciente integración y avance de la petroquímica básica fueron abandonados. En distinto grado, se incrementó la presencia del capital extranjero, las firmas sobrevivientes se concentraron fuertemente y se amplió el rezago tecnológico del sector respecto a la frontera internacional.

\section{La petroquímica básica en Argentina y México}

De acuerdo con la información disponible, realizaremos a continuación una comparación entre la evolución de la producción y el mercado petroquímico en Argentina y México durante el período en que la in- 
dustria estuvo motorizada por las empresas públicas:Yacimientos Petrolíferos Fiscales (YPF) y la Dirección General de Fabricaciones Militares (DGFM), en el primer caso; y Petróleos Mexicanos (PEMEX), en el segundo. Prestaremos especial atención a los seis productos primarios de las tres cadenas petroquímicas fundamentales, ya que en ellos se centró tanto la regulación como la acción estatal directa durante el período de sustitución de importaciones en Latinoamérica. Esos bienes eran amoníaco y metanol (cadena del metano), el etileno (olefinas) y benceno, tolueno y xilenos (BTX, aromáticos). De estos grupos se desprendían cientos de derivados, con destino prácticamente en todos los sectores industriales: plásticos, fibras sintéticas, fertilizantes, caucho, farmacéutica, adhesivos, solventes, aislantes, pinturas, plastificantes, explosivos e infinidad de otros subproductos químicos. En una enumeración no exhaustiva, para el caso de la primera cadena mencionada, su principal aplicación era la producción de fertilizantes, en el segundo la fabricación de plásticos - tanto rígidos como maleables - y en el último, para la obtención de fibras sintéticas.

En primer lugar, si bien en Argentina la fabricación de productos se dio de manera más temprana y diversificada desde comienzos de la década de 1940 (Tabla 4), el crecimiento posterior de la industria mexicana sobrepasó en gran medida el volumen de su producción petroquímica. En los inicios del verdadero despliegue a gran escala en los dos países, a principios de la década de 1960, ya México más que duplicaba el valor total del sector en Argentina (Tabla 5), y la diferencia continuó acrecentándose en las siguientes décadas. Así, para 1990 - cuando ya estaba en marcha el proceso de reversión de la estrategia de industrialización - la producción mexicana, de 14,5 millones de toneladas, representaba un monto de más de cinco veces la de su equivalente argentina.

Más allá de las diferencias en la disponibilidad natural de insumos hidrocarburíferos (mucho mayor en México), también jugó un papel fundamental la regulación estatal del sector y el accionar de las empresas públicas involucradas en la petroquímica. En México, si bien la industria petrolera se había nacionalizado en 1938, recién hacia 1960 se terminó de fijar el funcionamiento que debía tener PEMEX y las empresas privadas en la industria ${ }^{22}$. A partir de allí, la petrolera estatal mostró una

22 Las medidas más importantes en este sentido fueron la sanción de una nueva 
tracción preponderante sobre la petroquímica mexicana, que tuvo un auge fenomenal con el boom petrolero de finales de la década de 1970. En Argentina, más allá de que las primeras fábricas fueron instaladas por YPF y la DGFM en la década de 1940, luego se impidió que las empresas públicas participaran de nuevas actividades. La promoción "desarrollista" de 1958 se focalizó, a diferencia del esquema legal mexicano, en impulsar al capital privado, y en particular extranjero, que condujo a la conformación del primer polo petroquímico integrado del país en San Lorenzo (Provincia de Santa Fe) por parte de un consorcio estadounidense. Recién hacia 1970 se les permitió encarar nuevos proyectos aYPF y DGFM, que se vieron materializados en los complejos petroquímicos de La Plata y Bahía Blanca, ambos en la Provincia de Buenos Aires ${ }^{23}$. Sin embargo, a partir de la segunda mitad de la década de 1980 comenzó a dibujarse un nuevo rumbo en los dos países: las empresas estatales perdieron potencia y los nuevos esquemas legales tendieron, de manera creciente, hacia la privatización y la desregulación de la industria petroquímica ${ }^{24}$.

Tabla 4 - Año de inicio de la producción de petroquímicos básicos en Argentina y México

\begin{tabular}{|c|c|c|c|}
\hline Producto & FAMILIA & ARgentina & MÉXICO \\
\hline Amoníaco & \multirow[t]{2}{*}{ Metano } & 1951 & 1951 \\
\hline Metanol & & 1949 & 1969 \\
\hline Etileno & Olefinas & 1963 & 1966 \\
\hline Benceno & \multirow[t]{5}{*}{ Aromáticos } & 1946 & 1964 \\
\hline Tolueno & & 1946 & 1964 \\
\hline o-Xileno & & 1946 & 1964 \\
\hline p-Xileno & & 1946 & 1973 \\
\hline Xilenos mezcla & & 1946 & 1965 \\
\hline
\end{tabular}

Fuente: elaboración propia en base a Castañeda Ramirez (2003), Silvetti (1999), PEMEX, Anuarios Estadísticos.

"Ley Reglamentaria del Artículo 27 Constitucional en el Ramo del Petróleo" en noviembre de 1958, su "Reglamento" en agosto del siguiente año y el "Acuerdo" sobre la elaboración de petroquímicos básicos de abril de 1960; al respecto, puede verse García Lizama (1968).

Sobre los tres emprendimientos, puede consultarse Odisio $(2010,2014,2008)$ respectivamente.

24 Un estudio complementario a este, enfocado específicamente a los cambios de la regulación y el accionar de las empresas públicas entre las décadas de 1940 a 1990 en Argentina y México, es Odisio (2018). 
Tabla 5 - Producción petroquímica total en México y Argentina, 1960-1990 (millones de toneladas y crecimiento anual acumulativo promedio por década)

\begin{tabular}{l|c|c|c|c|c|c|c|c}
\hline & 1960 & 1970 & $\begin{array}{c}\text { CREC. ANUAL } \\
1960-1970\end{array}$ & 1980 & $\begin{array}{c}\text { CREC. ANUAL } \\
1970-1980\end{array}$ & 1990 & $\begin{array}{c}\text { CREC. ANUAL } \\
1980-1990\end{array}$ & $\begin{array}{c}\text { CREC. ANUAL } \\
1960-1990\end{array}$ \\
\hline PEMEX & 0,05 & 1 & $35 \%$ & 3,4 & $13 \%$ & 7,3 & $8 \%$ & $18 \%$ \\
\hline $\begin{array}{l}\text { Sector } \\
\text { secundario }\end{array}$ & - & 0,5 & - & 2,6 & $18 \%$ & 7,2 & $11 \%$ & $14 \% \star$ \\
\hline Total MX & 0,05 & 1,5 & $41 \%$ & 6 & $15 \%$ & 14,5 & $9 \%$ & $21 \%$ \\
\hline Total AR & 0,02 & 0,6 & $41 \%$ & 1,3 & $8 \%$ & 2,6 & $7 \%$ & $18 \%$ \\
\hline
\end{tabular}

Fuente: elaboración propia en base a estimaciones de Montaño Aubert (2001), San Martín (2006), Instituto Petroquímico Argentino, Anuario Estadístico.

* En este caso se trata del crecimiento medio anual tomando como año base 1970 (ya que en 1960 no se contaba con producción significativa del sector privado).

Ilustración 2 - El mercado petroquímico en Argentina, 1965-1990

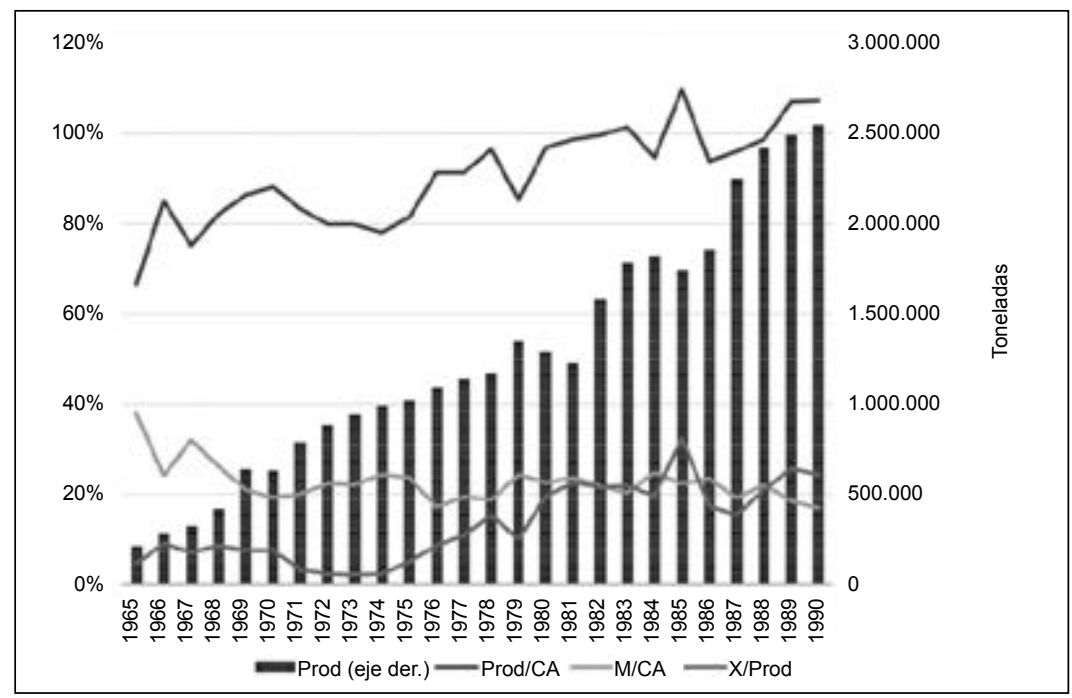

Fuente: elaboración propia en base a datos de San Martín (2006), Instituto Petroquímico Argentino, Anuario Estadístico.

Analizando la evolución del mercado de petroquímicos en Argentina, se percibe un crecimiento paralelo la expansión de la capacidad productiva y la demanda interna. Así, la relación entre producción y consumo aparente pasó de menos del 70\% a mediados de la década de 1960 a lograr satisfacer la totalidad del mercado hacia finales de la década siguiente (Ilustración 2). La producción total superó el medio millón de toneladas 


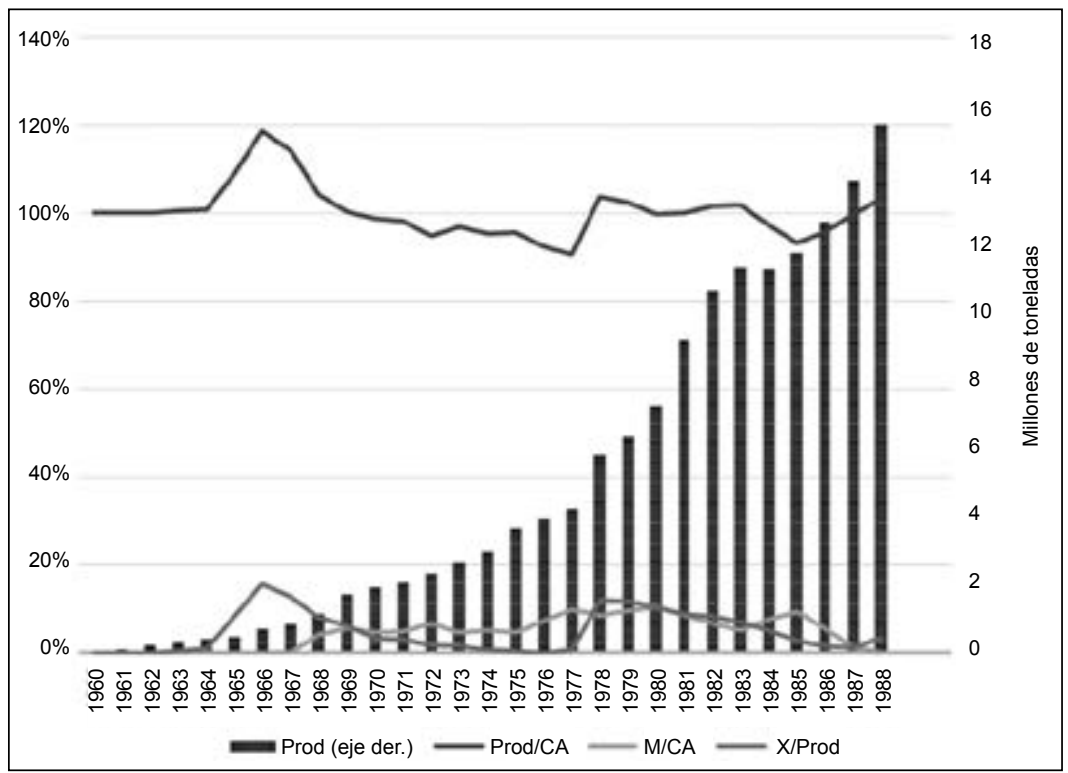

Fuente: elaboración propia en base a datos de PEMEX, Anuarios Estadísticos.

* A partir de 1989 los Anuarios cambiaron la forma de presentar los datos, pasando a informar únicamente la producción destinada a ventas (dejando fuera del volumen de producción lo destinado a autoconsumo).

a finales de la década de 1960, el millón un lustro después y los dos millones a finales de la década de 1980. Si bien las importaciones no lograron reducirse, manteniéndose en torno al 20\% del consumo aparente interno, las exportaciones si mostraron un progresivo avance, más que duplicando la participación de las ventas al exterior en el total de la producción petroquímica nacional entre 1965 y 1990.

En México, como dijimos, la expansión fue mucho más acusada. A fin de mantener un punto de comparabilidad entre los dos casos, consideraremos las variables cuantitativas referidas específicamente a PEMEX, que presenta su información también en toneladas. La producción total superó el millón de toneladas en 1968, diez años después alcanzó los cuatro millones y llegó superar los quince millones en 1988. En este caso, la producción antecedió la expansión del mercado; desde su comienzo y hasta finales de la década de 1960 el volumen producido se equiparó y excedió el nivel de absorción interno. En la década siguiente el crecimiento de PEMEX no alcanzó a mantener el ritmo de la 
demanda, cayendo hasta el 90\% en 1977. La fuerte expansión posterior permitió recuperar el abastecimiento. Con todo, durante todo el período las importaciones se mantuvieron en un nivel inferior al 10\% del consumo aparente. Las exportaciones mostraron la evolución típica de la industria petroquímica, con saltos en las ventas externas frente a los incrementos modulares de la capacidad productiva (1965-1969 y 19781984), luego atenuados por el crecimiento, más lineal, de la absorción interna.

Como señalamos más arriba, las importaciones tuvieron mayor preponderancia en el abastecimiento del mercado interno, mientras que las exportaciones se vieron más rezagadas (Ilustración 4). Por tal motivo, la balanza exterior mostró importantes resultados negativos, especialmente durante la década de 1970. En la década siguiente se presentaron algunos pocos años superavitarios (1983, 1985, 1989, 1990), los que respondieron fundamentalmente a crisis del mercado industrial interno, las que ocasionaban caídas importantes en la demanda por petroquímicos extranjeros.

Ilustración 4 - Saldo externo petroquímico en Argentina, 1965-1990

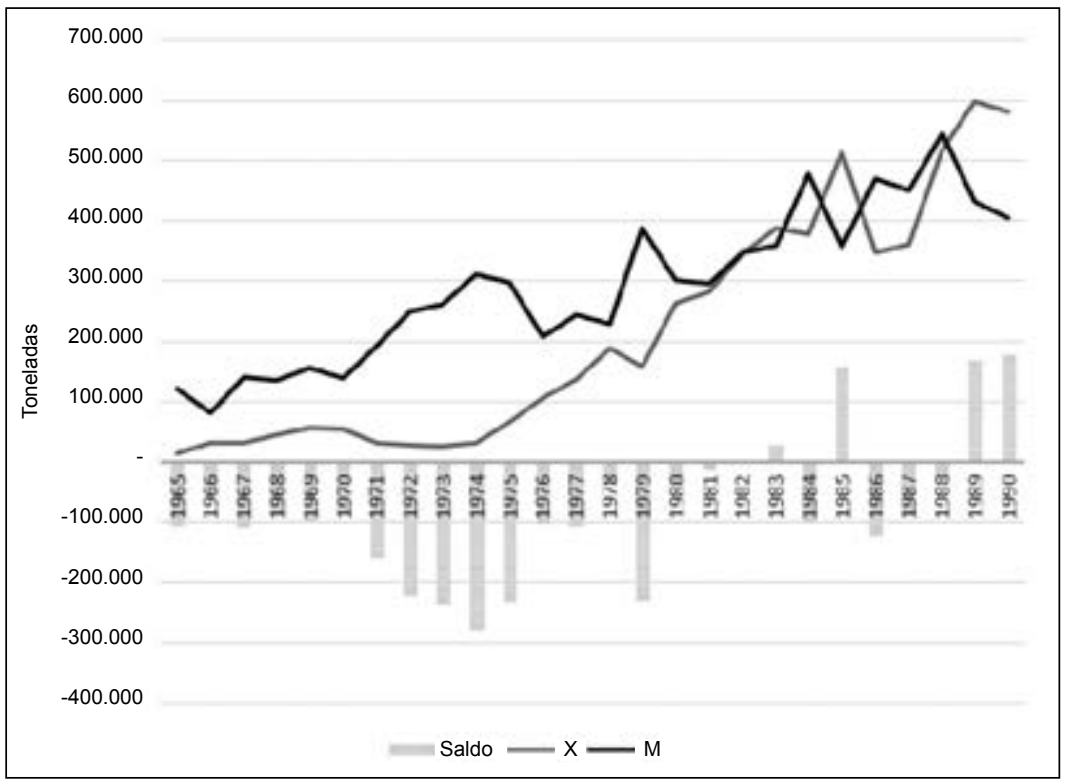

Fuente: elaboración propia en base a datos de Instituto Petroquímico Argentino, Anuario Estadístico. 
Si se considera el comercio exterior del sector petroquímico en México también puede observarse una relevancia persistente del deficit sectorial (Ilustración 5). Si esta situación contrasta con lo arriba mencionado acerca del mercado petroquímico se debe a que aquí se está tomando en consideración el total de la industria, y no solamente la situación asociada a PEMEX, que mostró mejores resultados externos que el resto del sector. De tal modo, durante las primeras dos décadas se pudo observar una creciente brecha comercial: mientras las importaciones pasaron de algo más de 100 millones de dólares en 1960 a 1.300 millones en 1981, recién hacia 1978 las exportaciones lograron sobrepasar los 100 millones de dólares, mostrando tanto un nivel menor como una expansión más lenta que las compras externas en todo el período.

Ilustración 5 - Saldo externo petroquímico en México, 1965-1990

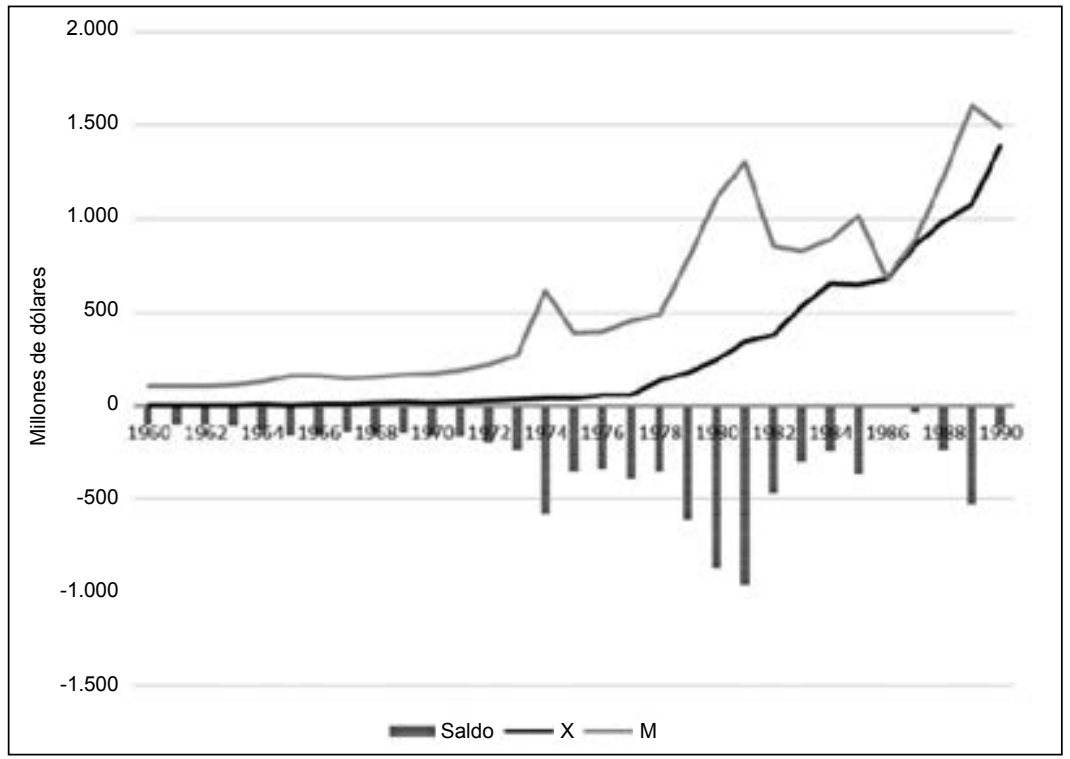

Fuente: elaboración propia en base a datos de Castañeda Ramirez (2003, Cuadro 11A).

Ahora bien, si centramos la atención en los seis productos básicos arriba mencionados, se puede observar que durante la década de 1970 la producción argentina se ubicó entre el 20\%-25\% del total mexicano (Ilustración 6).A partir de 1978 se produjo una mayor diferencia, porque 
en uno y otro país la estrategia divergió por esos años: mientras en Argentina comenzaba a transitarse un modelo de desindustrialización selectiva, retiro del Estado y fuerte apertura económica tras el arribo de la última dictadura cívico-militar al gobierno en marzo de 1976, en México el descubrimiento de ingentes reservas petroleras daba aire para una renovada y amplia expansión industrial y de las empresas públicas. Ese salto motivó que la suma del amoníaco, metanol, etileno y BTX producidos en Argentina disminuyera a un 15\% del nivel alcanzado por la industria mexicana.

Ilustración 6 - La petroquímica básica en Argentina vis a vis México, 1970-1990

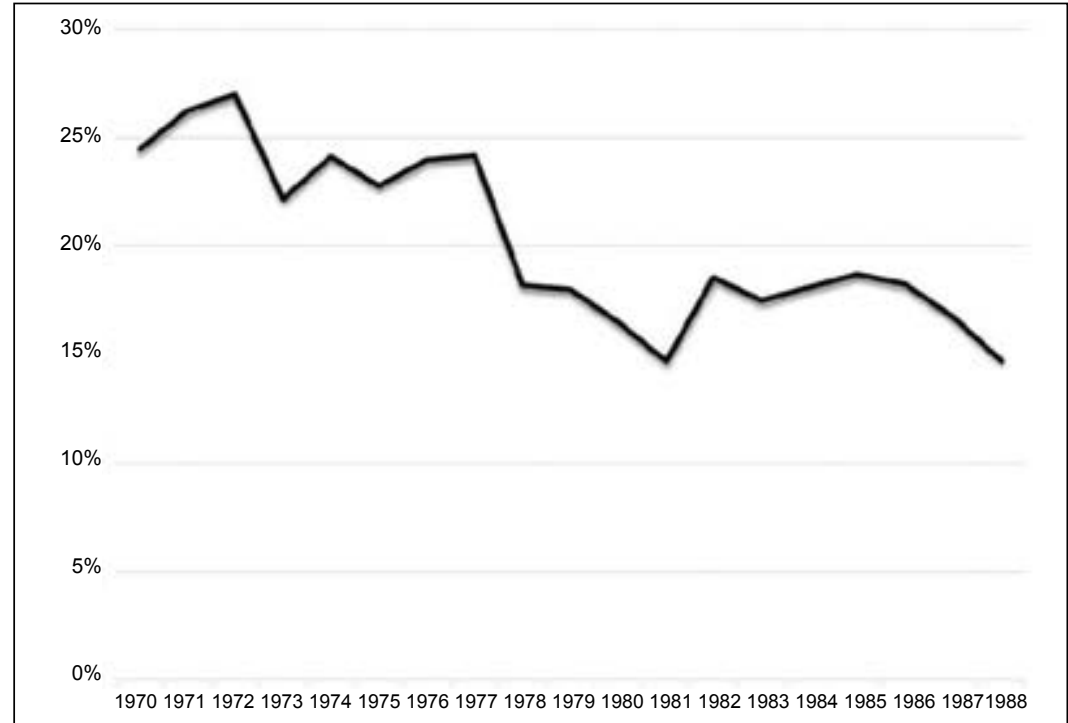

Fuente: elaboración propia en base a datos de PEMEX, Anuarios Estadísticos, Instituto Petroquímico Argentino, Anuario Estadístico.

En la industria petroquímica argentina los seis insumos fundamentales fueron representando una porción creciente sobre el total producido en el país. Desde un mínimo del 23\% en 1973 se llegó a ubicar en torno al 40\% a finales de la década de 1980 (Ilustración 7 - Petroquímicos básicos en Argentina, 1970-1990Ilustración 7). Con mayor detenimiento, se puede notar que el grupo más favorecido resultó el de aromáticos (benceno, tolueno, xilenos), que había recibido notable impulso en 1964 
con la puesta en funciones de los mencionados polos de San Lorenzo y de La Plata. La cadena del etano sólo tomó impulso en la década de 1980, con la puesta en funciones del mayor complejo en la Argentina: la Petroquímica Bahía Blanca. Dejamos aquí simplemente anotado que el grupo productivo del metano se volvió muy relevante en los últimos lustros, fuera ya de nuestro período de análisis.

Ilustración 7 - Petroquímicos básicos en Argentina, 1970-1990

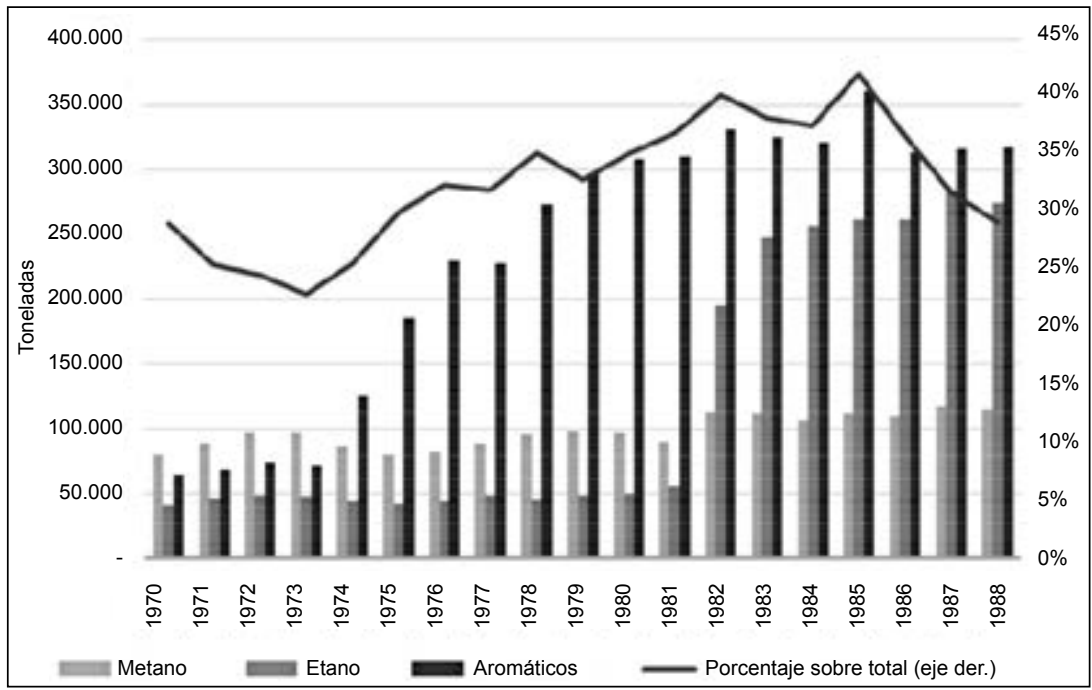

Fuente: elaboración propia en base a datos de Instituto Petroquímico Argentino, Anuario Estadístico.

En el caso mexicano también estos productos acapararon una porción sustantiva de la producción total aunque con una evolución distinta: desde un máximo del 52\% en 1965 cayó hasta niveles cercanos al 30\% del total a finales de la década de 1980 (Ilustración 8) ${ }^{25}$. Empero, una

25 Como no hemos podido reconstruir la serie histórica de la producción en valores físicos para todo el sector petroquímico mexicano, las cifras corresponden a la evolución productiva de PEMEX exclusivamente. En tanto la regulación desde la década de 1950 resguardó para Petróleos Mexicanos la fabricación de las materias primas que aquí se toman en cuenta, consideramos que - con las precauciones que corresponda tomar - su evolución es lo suficientemente representativa del total. Los cambios en los petroquímicos considerados "básicos" por la legislación mexicana se encuentran resumidos en Chow Pangtay (2002, Cuadro 25). 
diferencia más contrastante con el caso argentino se refiere a la importancia que adquirieron los derivados del metano. La petroquímica básica se enfocó fundamentalmente a la obtención de materias primas para fertilizantes; en promedio, el 65\% de la producción de básicos la acaparó el metanol y el amoniaco. Así, una más estrecha vinculación de la estrategia de industrialización con el desarrollo agrícola agregó una característica particular al proceso económico en México durante el siglo pasado ${ }^{26}$.

Ilustración 8 - Petroquímicos básicos en México (PEMEX), 1960-1988

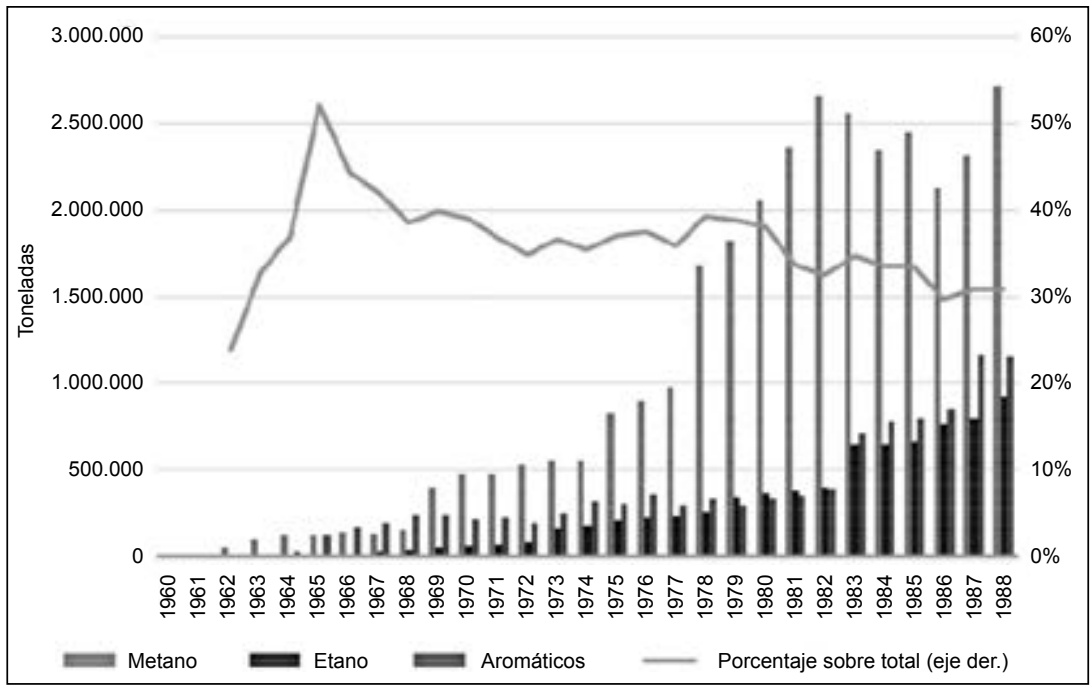

Fuente: elaboración propia en base a datos de PEMEX, Anuarios Estadísticos.

\section{Conclusiones}

En Argentina y México la producción petroquímica fue impulsada por el Estado desde sus inicios en las décadas de la segunda posguerra. Sin embargo, como hemos visto, la expansión fue en el segundo país

26 Este es un rasgo central del proceso de industrialización mexicano y si bien aparece mencionado en la historiografía sobre el tema, prácticamente no hay trabajos que tomen esa articulación entre desarrollo agrario e industrial como su eje de investigación. Una importante excepción es Aboites (1989). 
mucho más importante a pesar de haber iniciado las actividades algunos años más tarde. En ese sentido, probablemente jugó a favor el hecho de que, desde sus comienzos, parte importante de la producción petroquímica - y, muy especialmente, la de insumos - fue reservada a Petróleos Mexicanos, mientras en Argentina se confió primero al capital privado extranjero y recién a finales de la década de 1960 se permitió el ingreso de las empresas públicas al sector. Considerando la capacidad productiva total, la articulación entre los actores en la industria petroquímica funcionó claramente mejor en el caso mexicano.

Con todo, el rezago argentino fue menor en lo tocante a los petroquímicos básicos que en el total del sector, manteniéndose una posición relativamente menos retrasada. En ese sentido, la aparición de los dos grandes polos estatales - General Mosconi y Bahía Blanca - permitieron mantener el paso frente al impresionante ritmo de expansión que la petroquímica mexicana mostró a partir de la década de 1960. Más allá de la evolución común, también se pueden identificar algunas aristas diferentes para cada para caso: en Argentina se otorgó preponderancia a los petroquímicos aromáticos, mientras en México la mayor importancia recayó fundamentalmente en el amoniaco. Por otra parte, las importaciones satisficieron una porción mayor del mercado interno durante todo el período, pero también las exportaciones fueron más relevantes en relación al total producido. Por último, también fue sincrónica en los dos países - aunque no casualmente - la paulatina salida del Estado como regulador y actor en el entramado productivo de la petroquímica durante la década de 1980. La implementación de políticas aperturistas y de desregulación implicó la modificación en el funcionamiento de todo el sector que motivó, entre otros cambios, una creciente extranjerización y una expansión productiva más lenta.

Todo ello habilita a pensar que la estrategia general relativa a la política económica de la industrialización - en su nacimiento, despliegue y clausura - más allá de motivaciones y características particulares en cada caso, obedeció a un contexto mayor que les dio sustento común durante las décadas de posguerra. Por otra parte, los distintos ritmos y formas de implementación de la política sustitutiva se dieron seguramente asociadas a la particular situación y evolución de cada sociedad. Como cierre, es interesante notar que el consenso teórico, las acciones de los actores globales y el funcionamiento de la economía internacional dieron un 
giro copernicano entre 1950 y 1990: entre las condiciones que permitieron un decidido fomento a la integración industrial como forma de alcanzar el anhelado desarrollo económico hasta su posterior abandono en favor de la "liberalización" de las fuerzas del mercado cuatro décadas más tarde se ubicó el devenir - repasado aquí - de la petroquímica básica en México y Argentina.

\section{Referencias bibliográficas}

ABOITES, Jaime. Industrialización y desarrollo agrícola en México. México (DF): Plaza y Valdés/UAM, 1989.

AUTY, Richard. The product life-cycle and the location of the global petrochemical industry after the second oil shock. Economic Geography. Worcester (MA), v. 60, n. 4, p. 325-338, 1984.

BERTI, Eduardo. La petroquímica. Desarrollo y métodos. Buenos Aires: Esso SAPA, 1963. CASTAÑEDA RAMIREZ, Rosaura. La evolución de la industria petroquímica mexicana, 1943-1999. México (DF): UNAM, 2003 (Tesis de Licenciatura en Economía).

CAVANNA, Luis. Definición e importancia de los principales productos petroquímicos. Tendencias actuales y futuras de la producción petroquímica. En: Cursos sobre aspectos técnico-económicos de la industria petroquímica. Buenos Aires: Instituto Argentino del Petróleo/Asociación Química Argentina/Asociación Argentina de Ingenieros Químicos, 1968.

CEPAL. Las industrias petroquímicas en América Latina. SEMINARIO SOBRE EL DESARROLLO DE LAS INDUSTRIAS QUÍMICAS EN AMÉRICA LATINA, 1965, Caracas, ONU. Disponible en <http://repositorio.cepal.org/handle/11362/17875>. Accedido el 4 de julio de 2012.

CEPAL. La industria petroquímica en América Latina. Santiago de Chile: Consejo Económico y Social, ONU, 1966. Disponible en <http://repositorio.cepal.org//handle/11362/18215>. Accedido el 12 de junio de 2012.

CHAPMAN, Keith. The international petrochemical industry: evolution and location. Oxford: Blackwell Publishing, 1991.

CHÁVEZ AGUILAR, María Luisa. La petroquímica básica y su importancia en la economía nacional. México (DF): UNAM, 1974 (Tesis de Licenciatura en Economía).

CHOW PANGTAY, Susana. Petroquímica y sociedad. México (DF): Fondo de Cultura Económica, 2002.

CHUDNOVSKY, Daniel; LÓPEZ, Andrés. Auge y ocaso del capitalismo asistido. La industria petroquímica en América Latina. Buenos Aires: CEPAL/IDRC/Alianza, 1997.

CHUDNOVSKY, Daniel; LÓPEZ, Andrés; PORTA, Fernando. Ajuste estructural y estrategias empresariales en Argentina, Brasil y México. Un análisis comparativo de la in- 
dustria petroquímica y de máquinas herramientas. Buenos Aires: DT 11-CENIT, 1993.

DOAN, Herbert. Challenges in petrochemicals. Financial Analysts Journal. Charlottesville (VA), v. 23, n. 4, p. 35-37, 1967.

FAN, Joseph. Price uncertainty and vertical integration: an examination of petrochemical firms. Journal of Corporate Finance, n. 6, p. 345-376, 2000.

FIRPO, Francisco. Panorama internacional de la industria petroquímica y relación de nuestro desarrollo petroquímico con el de la ALALC. En: Cursos sobre aspectos técnico-económicos de la industria petroquímica. Buenos Aires: Instituto Argentino del Petróleo/Asociación Química Argentina/Asociación Argentina de Ingenieros Químicos, 1968.

GARCÍA LIZAMA, Martín. La industria petroquímica en México (reglamentación legal, permisos y empresas). México (DF): UNAM, 1968 (Tesis de Licenciatura en Derecho).

GARRITZ CRUZ, Andoni. Historia y prospectiva de la industria petroquímica y petrolera: relación con el tipo de cambio y otras variables macroeconómicas. México (DF): UNAM, 1996 (Tesis en Ingeniería Química).

GRIONI, Eradio.Valor económico de las materias primas frente a los productos químicos derivados. En: Cursos sobre aspectos técnico-económicos de la industria petroquímica. Buenos Aires: Instituto Argentino del Petróleo/Asociación Química Argentina/ Asociación Argentina de Ingenieros Químicos, 1968.

GUGLIELMO, Raymond. La petroquímica en el mundo. Buenos Aires: EUDEBA, 1960.

INSTITUTO Petroquímico Argentino, Anuario Estadístico. Buenos Aires: Instituto Petroquímico Argentino, varios años.

INSTITUTO Venezolano de Petroquímica. La industria petroquímica y su desarrollo en Venezuela. Caracas: Consejo Económico y Social, ONU, 1964.

KHANNA, Sushil. Transnational corporations and technology transfer: contours of dependence in Indian petrochemical industry. Economic and Political Weekly. Mumbai, v. 19, n. 31-33, p. 1.319-1.340, 1984.

MONTAÑ AUBERT, Eduardo. Integración de la petroquímica en México. México (DF): UNAM, 2001.

ODISIO, Juan. El complejo petroquímico de Bahía Blanca: una historia sinuosa. Estudos Ibero-Americanos. Porto Alegre, v. 34, n. 2, p. 114-129, 2008.

ODISIO, Juan. Febo asoma. Breve historia del polo petroquímico de San Lorenzo. Anuario CEEED. Buenos Aires, año 2, n. 2, p. 192-231, 2010.

ODISIO, Juan. Empresas públicas e industrialización. Petroquímica General Mosconi y el papel del Estado argentino en el desarrollo de la industria básica, 1969-1993. Buenos Aires: UBA, 2014 (Tesis de Doctorado en Ciencias Sociales).

ODISIO, Juan. Empresas del Estado y petroquímica en México y Argentina durante la industrialización por sustitución de importaciones. Signos Históricos. México (DF), v. 20, n. 40, p. 262-311, 2018.

PEMEX. Anuario Estadístico. México (DF): Petróleos Mexicanos, varios años.

RODRÍGUEZ MAGALLANES, Diego. Orígenes, evolución y perspectivas de la industria petroquímica. México (DF): UNAM, 2008 (Tesis en Ingeniería Química). 
SAN MARTÍN, José. El petróleo y la petroquímica en la Argentina (1914-1983). Buenos Aires: Ediciones Cooperativas, 2006.

SILVETTI, Edgardo. La República Argentina y su industria petroquímica. Buenos Aires: Instituto Petroquímico Argentino, 1999. Disponible en <http://www.ipa.org.ar/ images/PUBLICACIONES/LaRepblicaArgentinaysuIndustriaPetroqumica.pdf $>$. Accedido el 30 de julio de 2012.

SPEIGHT, James. Petroleum and petrochemicals. In: ALI, Mohammad Farhat; EL ALI, Bassam; SPEIGHT, James (ed.). Handbook of industrial chemistry. Organic chemicals. New York: McGraw-Hill, 2005, p. 473-510.

STOBAUGH, Robert. The neotechnology account of international trade: the case of petrochemicals. Journal of International Business Studies. East Lansing (MI), v. 2, n. 2 , p. 41-60, 1971a.

STOBAUGH, Robert. The international transfer of technology in the establishment of petrochemical industry in developing countries. New York: UNITAR, 1971b (Research Report, 12). 